\section{Effect of topical bupivacaine on post-tonsillectomy pain relief in children}

\author{
Musa Kallamu Suleiman, ${ }^{1}$ Abdulkarim \\ Aitek Abdullahi, ${ }^{1}$ Alhassan Datti \\ Mohammed, ${ }^{2}$ Kufre Robert Iseh, ${ }^{3}$ \\ Abdulrahman Aliyu, ${ }^{1}$ Ibrahim \\ Galadima Bello \\ ${ }^{1}$ Department of Anaesthesiology and \\ Intensive Care, Usmanu Danfodiyo \\ University Teaching Hospital, Sokoto; \\ ${ }^{2}$ Department of Anaesthesiology and \\ Intensive Care, Aminu Kano Teaching \\ Hospital, Kano; ${ }^{3}$ Department of \\ Otorhinolaryngology, Usmanu \\ Danfodiyo University Teaching Hospital, \\ Sokoto, Nigeria
}

\begin{abstract}
Tonsillectomy is a common surgery performed in paediatric otorhinolaryngology and is usually accompanied by considerable postoperative pain. Earlier literature has shown the use of topical bupivacaine for post-tonsillectomy pain relief to be promising. This study was conducted to evaluate the efficacy of topical bupivacaine application on post-tonsillectomy pain relief. Fifty consenting patients scheduled for tonsillectomy that met the inclusion criteria were enrolled in the study and assigned into two groups of 25 patients each. Group B had their tonsillar fossa packed with $0.5 \%$ bupivacaine soaked gauze for 5 minutes while Group $\mathrm{S}$ had normal saline. Pain intensity was measured at 1, 2, 4, 12 and 24 hours postoperatively, using the Faces Pain ScaleRevised (FPS-R). Mean scores for groups B and $\mathrm{S}$ at 1, 2, 4, 8, 12, 24 hours were $1.96 \pm 1.17,2.40 \pm 0.82,2.8 \pm 1.0,2.88 \pm 1.17$, $3.08 \pm 0.99$, and $3.04 \pm 1.02$ and $3.36 \pm 1.38$, $4.72 \pm 1.62, \quad 3.92 \pm 1.35, \quad 3.76 \pm 1.45$, $4.00 \pm 1.41,3.38 \pm 0.98$ respectively. The difference was significant at 1 and 2 hours only $(\mathrm{P} \leq 0.05)$. Post-tonsillectomy pain was reduced in the first two hours by application of bupivacaine soaked gauze.
\end{abstract}

\section{Introduction}

Tonsillectomy can be defined as a surgical procedure performed with or without adenoidectomy in which the tonsils are completely removed, including its capsule. ${ }^{1}$ Essentially, it involves dissecting the peritonsillar space between the tonsils capsule and the muscular wall. It is one of the most commonly performed day-case surgeries in children in high-income countries and is usually accompanied by moderate to severe postoperative pain. ${ }^{1,2}$

Managing post tonsillectomy pain remains a challenge to both the anaesthetist and the surgeon, as inadequate pain management often leads to delayed discharge, unplanned readmissions, dehydration, infection and secondary haemorrhage. ${ }^{1-3}$ Different surgical and anaesthetic techniques have been developed for use during and after the surgery to reduce post-tonsillectomy pain, some of which have shown some positive outcomes in randomised trials. $^{3,4}$

Opioids, Non-Steroidal AntiInflammatory Drugs (NSAID) and acetaminophen are used traditionally for posttonsillectomy pain relief. However, opioids can cause adverse effects such as sedation, nausea and vomiting, cough suppression, respiratory depression, while NSAIDs may increase bleeding tendencies. ${ }^{2,3,5}$ Because of the lack of these side effects in Local Anaesthetics (LAs), there has been a renewed interest in their use for post-tonsillectomy pain relief. Local anaesthetics are usually administered in three different ways for this purpose: i) re-incisional peritonsillar infiltration; ii) post-tonsillectomy wound infiltration; iii) post-tonsillectomy topical packing with soaked gauze.

Wound infiltration with bupivacaine has been reported to cause visual loss, ${ }^{6}$ cervical osteomyelitis $^{7}$ and airway obstruction. ${ }^{8}$ Also, if inadvertently injected into a blood vessel, bupivacaine can result in cardiac arrhythmia, which is difficult to treat. However, various studies have used bupivacaine soaked gauze without any serious complication reported. $2,3,5,9$

This study aims to evaluate the efficacy of topical application of bupivacaine on post-tonsillectomy pain relief in children. The primary outcome is the FPS-R scores of the two groups, while the secondary outcomes are the time to first analgesic request, and the cumulative dose of systemic analgesics consumed.

\section{Materials and Methods}

This is a prospective double-blinded randomised controlled study of 50 ASA I and II patients aged between 5 to 15 years scheduled for elective tonsillectomy in Usmanu Danfodiyo University Teaching Hospital (UDUTH), Sokoto, Nigeria, between $1^{\text {st }}$ August 2017 to $1^{\text {st }}$ January 2018. After approval by the Research and Ethics Committee of the hospital, informed consent was obtained from all patients after a thorough explanation of the purpose and
Correspondence:Alhassan Datti Mohammed, Department of Anaesthesiology and Intensive Care, Aminu Kano Teaching Hospital/Bayero University, Kano, Nigeria.

Tel.: +234 8069297557

E-mail: dradmoh3@gmail.com

Key words: Bupivacaine; pain-relief; posttonsillectomy; children.

Contributions: MKS was the principal investigator in this study from proposal, design and protocol development through data analysis to the final manuscript writing. AAA contributed in the review of the article. ADM contributed in the study design, manuscript writing and review of the article. KRI contributed in the design and review of the article. AA supported in the study design, data analysis and contributed in the review of thearticle. IMG contributed in the draft and review of the article. All authors have read and agreed to the final version of this manuscript.

Conflict of interest: The authors declare no conflict of interest.

Ethics approval and consent to participate: The study was approved by the Research and Ethics Committee of the hospital. Informed consent was obtained from all patients after a thorough explanation of the purpose and scope of the study before the commencement of the study.

Consent for publication: Patients' guardians gave their consent for publication.

Availability of data and materials: All data generated or analyzed during this study are included in this published article.

Received for publication: 8 September 2019. Revision received: 27 October 2019.

Accepted for publication: 27 October 2019.

This work is licensed under a Creative Commons Attribution NonCommercial 4.0 License (CC BY-NC 4.0).

CC Copyright: the Author(s), 2020

Licensee PAGEPress, Italy

Pyramid Journal of Medicine 2020; 3:64 doi:10.4081/pjm.2020.64

scope of the study before the commencement of the study.

Patients whom parents/guardian were not willing to participate, patients with proven or suspected allergy to local anaesthetics, patients for combined adenotonsillectomy, patients with peritonsillar abscess or suspected tonsillar malignancy, patients with difficulty in understanding the Faces Pain Scale-Revised (FPS-R) and sickle cell disease patients were excluded from the 
study. Routine laboratory investigations including full blood count, Serum electrolytes, urinalysis, and when indicated Electrocardiograph (ECG), chest radiograph and coagulation studies were conducted. The patients were randomly assigned using a sealed envelope technique into two groups (Group B and S) of 25 each in a double-blinded fashion. All patients were anaesthetised using standard protocol. Atropine and dexamethasone were given as premedicants to both groups. General anaesthesia was induced with i.v. propofol, fentanyl and suxamethonium to facilitate orotracheal intubation. Correct tube placement was confirmed by capnography and chest auscultation. Anaesthesia was maintained with isoflurane and $100 \%$ oxygen to maintain adequate anaesthetic depth. Muscle relaxation was maintained with topups of atracurium while intraoperative analgesia was achieved with fentanyl. The oxygen saturation, pulse rate, blood pressure, $\mathrm{ETCO}_{2}$ and airway pressure were continuously monitored intraoperatively. Blood loss was assessed by counting the number of pieces of gauze used and estimating the amount of blood in the suction bottle. Isoflurane was discontinued at the end of the surgery, and fresh gas flow increased to 4-6 L/min. Atropine and neostigmine were used to reverse the residual effect of muscle relaxant.

Two surgeons adjudged to be of equal proficiency performed the surgeries using a standardised cold knife dissection technique

After haemostasis was achieved, group $B$ patients had both sides of their tonsillar fossae packed with standard gauze of $10 \mathrm{~cm}$ dimension folded twice to make it 2.5 by $2.5 \mathrm{~cm}$ and fully soaked with $5 \mathrm{ml}$ of $0.5 \%$ bupivacaine (Marcaine AstraZeneca brand). The pack was removed after applying five minutes of firm pressure. In group $S$ patients, bupivacaine was replaced by normal saline, and saline-soaked gauze was applied in the same fashion as applied in Group B.

The pain intensity (FPS-R score) of each patient was assessed and recorded by the researcher and another anaesthetist who is conversant with FPS-R at 1, 2, 4, 8, 12 and 24 hours postoperatively. The time to first analgesic request defined as the time between the end of surgery and the first dose of diclofenac $(0.5 \mathrm{mg} / \mathrm{kg})$ administered was recorded for each patient. Henceforth, patients were placed on diclofenac every $12 \mathrm{hrs}$. For breakthrough pains (FPS-R score $>4$ ), rescue analgesia was administered and recorded as a repeated dose of intravenous diclofenac. After 24 hours, the cumulative dose of diclofenac consumed was recorded, and patients were converted to oral ibupro- fen $5 \mathrm{mg} / \mathrm{kg} / \mathrm{dose}$ and oral acetaminophen $15 \mathrm{mg} / \mathrm{kg}$ every 8 hours

Only data obtained from patients who were well oriented in person, place and time were considered for statistical analysis. Statistical analysis of data collected was performed using SPSS version 20.0. Results were expressed as the mean $\pm \mathrm{SD}$ except where stated otherwise. Differences in FPS$\mathrm{R}$ scores between the two groups were evaluated with Student's $t$-tests. The time to first analgesic request and cumulative dose of diclofenac consumed were analysed with the unpaired Student's t-test after logarithmic transformation to ensure a normal distribution. A P value $<0.05$ was considered significant.

\section{Results}

The two (2) groups consisting of 25 patients each. Group B had their tonsillar fossae packed with $0.5 \%$ bupivacaine soaked gauze while group $\mathrm{S}$ had their tonsillar fossae packed with saline-soaked gauze.

Table 1 shows the demographic profile of the patients. Both groups were comparable in terms of age, sex, weight and ASA classification distribution. Group B has a mean age of $8.0( \pm 3.06)$ years, while that of group $\mathrm{S}$ is $9.48( \pm 3.12)$ years.

The percentage of male patients in both groups is $9(36 \%)$ and 11 (44\%) for group B and $\mathrm{S}$ respectively giving a total of 20 $(40 \%)$ male patients who participated in the study. Female patients who participated in the study were found to be $16(64 \%)$ and 14 (66\%) for group B and S respectively, making a total of $30(60 \%)$ female patients, (pvalue $=0.305$ ). The patients in both groups were also comparable in weight distribution with a mean weight of $28.84( \pm) \mathrm{kg}$ for group B and $30.32( \pm) \mathrm{kg}$ for group $\mathrm{S}$ with a p-value of 0.26 . The ASA physical status classification distribution revealed $9(36 \%)$ in group B and $8(32 \%)$ in group S for ASA I, while for ASA II 16 (64\%) were from group B and 17 (68\%) from group S.

Table 2 shows that FPS-R score at 1 and 2 hours postoperatively was significantly lower in Group B with a mean score of 1.96 $( \pm 1.17)$ and $2.40( \pm 1.00)$ compare to 3.36 $( \pm 1.38)$ and $4.72( \pm 1.62)$ for patients in group $\mathrm{S}$ with a p-value of 0.03 and 0.003 respectively. The table also shows the mean FPS-R score of group B patients to be lower than that of group $S$ throughout the period of the postoperative pain assessment.

Table 1. Demographic profile.

\begin{tabular}{lccc} 
Variables & $\begin{array}{c}\text { Bupivacaine group } \\
\mathbf{N}=25\end{array}$ & $\begin{array}{c}\text { Saline group } \\
\mathbf{N}=25\end{array}$ & P-value \\
$\begin{array}{l}\text { Age (years) } \\
\text { Mean }( \pm \text { SD) }\end{array}$ & $8.00(3.06)$ & $9.48(3.12)$ & 0.15 \\
$\begin{array}{l}\text { Weight }(\mathrm{kg}) \\
\text { Mean }( \pm \text { SD) }\end{array}$ & $28.84(7.12)$ & $30.32(5.48)$ & 0.26 \\
\hline $\begin{array}{l}\text { Sex }(\%) \\
\text { Male }\end{array}$ & $9(36)$ & $11(44)$ & \\
Female & $16(64)$ & $14(66)$ & \\
ASA status $(\%)$ & $9(36)$ & $8(32)$ & \\
I & $16(64))$ & $17(68)$ & \\
II & &
\end{tabular}

Table 2. Postoperative FPS-R Scores.

\begin{tabular}{|c|c|c|c|c|}
\hline Time & $\begin{array}{l}\text { Bupivacaine group } \\
\text { Mean }( \pm \mathrm{sd}) \\
\mathrm{N}=25\end{array}$ & $\begin{array}{l}\text { Saline group } \\
\text { Mean }( \pm s d) \\
N=25\end{array}$ & Mean difference & P-value \\
\hline $1^{\text {st }}$ hour & $1.96(1.17)$ & $3.36(1.38)$ & 1.40 & 0.034 \\
\hline $2^{\text {nd }}$ hour & $2.40(0.82)$ & $4.72(1.62)$ & 2.30 & 0.003 \\
\hline $4^{\text {th }}$ hour & $2.8(1.00)$ & $3.92(1.35)$ & 1.12 & 0.847 \\
\hline $8^{\text {th }}$ hour & $2.88(1.17)$ & $3.76(1.45)$ & 0.88 & 0.724 \\
\hline $12^{\text {th }}$ hour & $3.08(0.99)$ & $4.00(1.41)$ & 0.92 & $0.2^{18}$ \\
\hline 24th hour & $3.04(1.02)$ & $3.28(0.98)$ & 0.42 & 0.170 \\
\hline
\end{tabular}

p $>0.05$ not significant 
However, no statistically significant difference was found at 4, 8, 12 and 24 hours.

Table 3 shows a statistically significant increase in the time to first analgesic request (TFA) in group B, which was 9.04 $( \pm 4.89)$ hours, when compared to group $\mathrm{S}$ which had a mean duration of $3.72( \pm 2.85)$ hours, with a p-value $<0.001$.

The cumulative dose of diclofenac consumption (CDD) between the two groups also showed a statistically significant reduction in patients who had bupivacaine with a mean of $35.84( \pm 19.44) \mathrm{mg}$ while the saline group had a mean of $78.60( \pm 30.14) \mathrm{mg}(\mathrm{p}$ value $<0.001)$.

\section{Discussion}

Adequate postoperative pain relief is a cardinal part of standard anaesthetic practice. It is a fact that inadequate pain relief prolongs postoperative recovery and hospital stay, thereby increasing the cost of care. The above factors impact negatively on the patients and their families, especially in countries with developing economy. Following tonsillectomy, inadequate pain control leads to unwanted physiological response such as tachycardia which increases the stress on the cardiovascular system, especially in patients with obstructive sleep apnea who may have right heart strain. It may also lead to restlessness with the refusal of oral feeds, which results in dehydration, infection and secondary haemorrhage. ${ }^{5}$

Post-tonsillectomy pain relief is traditionally achieved with opioids and NSAIDs. However, their use is associated with complications such as respiratory depression, cough suppression, increased incidence of desaturation and increased postoperative bleeding (may be caused by NSAID). ${ }^{2,3,5,10}$ Kelly and colleagues, ${ }^{11}$ randomised 91 children aged 1 to 10 years to compare the effect and safety of Morphine and Ibuprofen for post-tonsillectomy analgesia. They found that only 14 per cent of patients in the morphine group had an improvement in their oxygen saturation compared to 68 per cent in the ibuprofen group $(\mathrm{p}<0.01)$. They also found that the number of desaturation events increased substantially in the morphine group $(\mathrm{p}<0.01)$. Therefore, they concluded that ibuprofen provides safe and effective analgesia in children undergoing tonsillectomy and that post-tonsillectomy use of morphine should be limited, as it may be unsafe in some children.

A Cochrane systematic review concluded that there is no sufficient evidence to exclude an increased risk of bleeding when NSAIDs is used in paediatric tonsillectomy. ${ }^{12}$ Hence, the need for alternative means of achieving post-tonsillectomy pain relief. This alternative should be used either solely or as a complementary agent, to reduce the dose requirement of opioids and NSAID, thereby minimising their side effects.

This study was intended to explore the effect of using topical bupivacaine as a complementary means of achieving posttonsillectomy pain relief and its effect on the systemic analgesic requirement in children.

Our study revealed the postoperative pain intensity to be significantly lower in the bupivacaine group when compared to the saline group with $\mathrm{p}=0.034$ and $\mathrm{p}<0.001$, at 1 and 2 hours, respectively. The difference in mean for the two groups 1.40 at 1 hour and 2.30 at 2 hours was the highest observed, which corresponds with the peak duration of action of bupivacaine. The lower values obtained in both groups at 1 hour postoperatively is likely due to the residual effect of the intraoperative analgesia. The peaking of the mean pain score of the saline group at the $2^{\text {nd }}$ hour can be attributed to the wearing out of the above factor. The subsequent $4^{\text {th }}, 8^{\text {th }}, 12^{\text {th }}$ and the $24^{\text {th }}$ hour, showed no significant difference between the two groups in terms of their FPS-R scores. The above can be attributed to the increasing pain scores in group B due to wearing out of bupivacaine's effect, and a relative decrease in pain scores of group $\mathrm{S}$ due to early commencement of diclofenac. At $24^{\text {th }}$ hour after surgery, lower pain scores were still observed in group B when compared to group $\mathrm{S}$, though it was not significant $(\mathrm{p}=0.173)$.

Table 3. Analgesic requirement pattern.

\begin{tabular}{|c|c|c|c|}
\hline Variables & $\begin{array}{l}\text { Bupivacaine } \\
\text { Group } \\
\text { Mean }( \pm \text { SD) } \\
n=25\end{array}$ & $\begin{array}{c}\text { Saline } \\
\text { Group } \\
\text { Mean ( } \pm \text { SD) } \\
n=25\end{array}$ & $\begin{array}{c}\text { Significance level } \\
\text { (p-value) }\end{array}$ \\
\hline TFA (hour) & $9.04(4.894)$ & $3.72(2.851)$ & 0.006 \\
\hline CDD (mg) & 35.84 (19.442) & $78.60(30.140)$ & 0.008 \\
\hline
\end{tabular}

$\mathrm{p}>0.05$ not significant. TFA: Time to first analgesic request. CDD: Cumulative dose of diclofenac consumed.

The above findings are similar to that of Feroz et al. ${ }^{13}$ However, it is worthy to note the relatively high pain score at 1 hour $(4.07 \pm 1.78)$ in their study as against that of ours. The above observation is likely due to the use of electrocautery, which is known to increase post-tonsillectomy pain. ${ }^{2}$ Also, the use of halothane to maintain anaesthesia, which takes a long time to clear when compared to the isoflurane used in this study, which clears faster. This might have undermined the patient's ability to report pain accurately just one hour after surgery. Therefore, as the residual effect of anaesthesia wears off, the patients became more aware and more of bupivacaine's effect on pain relief was observed.

Our findings are also similar to that of Sabbar et al. $^{2}$ who demonstrated that there is a significant reduction in pain scores (VAS) of patients who had $5 \mathrm{~mL}$ of $0.5 \%$ bupivacaine soaked gauze applied to their tonsillar bed after tonsillectomy when compared to the control group who had normal saline. The highest difference in mean pain score in our study was at 2 hours postoperatively, with a value of $2.30(\mathrm{p}=0.003)$, compares favourably with that of Sabbar's study $1.43(\mathrm{p}<0.001)$ suggesting a period of maximal effect of bupivacaine in both studies. However, there was no information on the intraoperative analgesic used in Sabbar's study, which might affect the interpretation of the study.

In contrast to our study, Sharma et al. ${ }^{9}$ found no statistically significant difference in the VAS scores between the bupivacaine group and the control group who had nothing. It can be argued that the unequal number of patients in the two groups (54 to 24) with the test group having more patients and the lack of a placebo, maybe the reasons why no statistically significant difference was found in the Sharma's study. Also, only two people were involved in pain assessment in our study, while the staff nurses on duty were used for pain assessment in Sharma's study. This translates into different people being involved in pain assessment, which may result in significant inter-observer variation affecting the results. ${ }^{14}$ Khan and colleagues ${ }^{15}$ also found no statistically significant difference in the VAS scores between the bupivacaine group and the saline group. Though their methodology was very similar to that of this study, they did not provide information on the mode of intraoperative analgesia administered, which might be responsible for the insignificant difference between the two groups. However, it is important to note that marginally lower pain scores were observed in the bupivacaine group when compared to the saline group. 
Our study was able to demonstrate a significant difference in duration before the first request for systemic analgesics in the bupivacaine group when compared to the saline group $p=0.006$. In agreement with the above finding Saki et al. ${ }^{16}$ also found the bupivacaine group to have spent longer time before requesting for analgesics $(\mathrm{P}=0.002)$ when compared to the placebo group which further shows the systemic analgesic sparing effect of topical bupivacaine after tonsillectomy surgery in children.

This study revealed that the bupivacaine group consumed fewer analgesics compared to the saline group $\mathrm{p}=0.008$ which is similar to the findings by Feroz et al. ${ }^{13}$ However, Sharma's study ${ }^{9}$ revealed no statistically significant difference between the two groups with regards to analgesic consumption. This contrasting finding can be attributed to the surprisingly higher pain score $(>7)$ that was set as cut off mark for rescue analgesic administration as against a pain score of $>4$ used in our study and by most other studies..$^{2-5,9,13,15,16}$ It is worthy to note that none of the patients in Group B had bupivacaine toxicity, which compares favourably with similar studies $2,4,5,9,16$ where bupivacaine soaked gauze was topically applied in the tonsillar fossa after tonsillectomy in children.

Overall this present study has been able to demonstrate an improvement in pain control with topical application of bupivacaine, similar to that found in other studies. ${ }^{2,4,13,16}$

\section{Conclusions}

We conclude that topical application of $0.5 \%$ bupivacaine is effective in reducing post-tonsillectomy pain in the first two hours after surgery and also reduces systemic analgesics requirement in children.

\section{References}

1. Mitchell RB, Archer SM, Ishman SL, et al. Clinical practice guideline: tonsillectomy in children (Update)-Executive summary. Otolaryngol Head Neck Surg 2019;160:187-205.

2. Sabbar S, Khan FA, Khalid A. The Effect of topical bupivacaine $0.5 \% 0 \mathrm{n}$ post-tonsillectomy pain. Med Channel 2009;15:15-7.

3. Bameshki AR, Razban M, Khadivi E, et al. The effect of local injection of epinephrine and bupivacaine on post-tonsillectomy pain and bleeding. Iran $\mathrm{J}$ Otorhinolaryngol 2013;25:209-13.

4. Ehsan-U1-Haq, Udaipurwala HI, Farrukh MS, Kumar H. Post-tonsillectomy pain reduction by application of bupivacaine pack in the tonsillar fossa. Pak J Surg 2009;25:76-9.

5. Hydri AS, Malik SM. Post-tonsillectomy pain and bupivacaine: An intra-individual design study. J Coll Physicians Surg Pak 2010;20:538-41.

6. Ellis P. Visual loss following tonsillectomy: Possible association with injections in tonsillar fossae. Arch Otolaryngol 1968;87:128-30.

7. Cyna A, Bell K, Flood L. Cervical osteomyelitis following tonsillectomy. Anaesth 1997;52:1084-7.

8. Bean-Lijewski JD. Glossopharyngeal nerve block for pain relief after pediatric tonsillectomy: Retrospective analysis and two cases of life-threatening upper airway obstruction from an interrupted trial. Anesth Analg 1997;84:1232-8.

9. Sharma S, Dave V, Zalawadia B, Shah $\mathrm{S}$. The effect of topical bupivacaine in post-tonsillectomy pain relief. GCSMC J Med Sci 2015;4:15-8.

10. Hydri AS, Nawaid MA, Afridi JA. Comparison of local application versus infiltration of bupivacaine for post-tonsillectomy pain in adults. Ann Pak Inst Med Sci 2012;8:3-5.

11. Kelly LE, Sommer DD, Ramakrishna J, et al. Morphine or Ibuprofen for posttonsillectomy analgesia: A randomized trial. Pediatr 2015;135:307-13.

12. Lewis SR, Nicholson A, Cardwell ME, et al. Nonsteroidal anti-inflammatory drugs and perioperative bleeding in paediatric tonsillectomy. Cochrane database Syst Rev 2013;7:CD003591.

13. Feroz S, Mahmood Z, Khatri F, et al. Can post-tonsillectomy pain be reduced by topical bupivacaine? A prospective randomized trial. Pak J Otolaryngol 2013;29:11-5.

14. Kouchi M, Mochimaru M, Tsuzuki K, Yokoi T. Interobserver errors in anthropometry. J Hum Ergol (Tokyo) 1999;28:15-24.

15. Khan MI, Shah SF, Muhammad, et al. Relief of post-tonsillectomy pain by topical application of bupivacaine. Khyber Med Univ J 2012;4:183-6.

16. Saki N, Jasemi NA, Mashali L, et al. Effect of topical bupivacaine on posttonsillectomy pain relief in children. Jundishapur J Nat Pharm Prod 2015;10:1-3. 